\title{
CARACTERIZACIÓN DE ACELGA FRESCA DE SANTIAGO DEL ESTERO (ARGENTINA). COMPARACIÓN DEL CONTENIDO DE NUTRIENTES EN HOJA Y TALLO. EVALUACIÓN DE LOS CAROTENOIDES PRESENTES ${ }^{1}$
}

\author{
Sara Macías de COSTA², Mariana A. MONTENEGRO², Teresa ARREGUI $^{3}$, \\ M. Inés Sánchez de PINTO², Mónica A. NAZARENO², Beatriz López de MISHIMA,*
}

\begin{abstract}
RESUMEN
Se realizaron estudios de caracterización integral de acelga (Beta vulgaris, var. cycla) cultivada en la Provincia de Santiago del Estero, Argentina. Por tratarse de un vegetal de importante consumo regional, se realizó la determinación de sus constituyentes básicos, minerales y pigmentos para conocer los valores nutricionales que aporta este alimento en nuestra región. Se analizaron comparando hoja y tallo: humedad, cenizas, proteinas, grasas, hidratos de carbono utilizables, fibra dietaria total, contenido de clorofila y carotenoides. En cuanto a minerales, se determinaron calcio, magnesio, sodio, potasio, manganeso, zinc, hierro, fósforo y boro. Se encontraron diferencias en los aportes de minerales, proteinas, grasas y fibra entre hoja y tallo, así como también el contenido de carotenoides exclusivamente en las hojas. Los resultados marcan importantes diferencias en el contenido energético y en su valor provitaminico A entre la hoja y el tallo de este vegetal, mostrando que este último es una parte aprovechable de la planta, recomendable en dietas hipocalóricas.
\end{abstract}

Palabras-clave: composición centesimal; minerales; carotenoides; provitamina A; acelga.

\section{SUMMARY}

CHARACTERIZATION OF FRESH Beta vulgaris FROM SANTIAGO DEL ESTERO (ARGENTINA).NUTRIENT AND CAROTENIOD CONTENT OF STEM AND LEAVES. An integrated characterization study of Swiss chard grown in Santiago del Estero, Argentina, was carried out. As Swiss chard is a vegetable of important regional consumption, the determination of its basic constituents, minerals and pigments was done to appraise the nutritional value offered by this food in our area. Moisture, ash content, proteins, fats, available carbohydrates, total dietary fiber, chlorophylls and carotenoids were analysed, comparing the leaves and the stems. As to minerals, calcium, magnesium, sodium, manganese, zinc, iron, phosphorous, potassium, and boron were determined. Differences in the concentration of minerals, proteins, fats and fiber between the leaves and the stems were found, as well as the carotenoid content mainly in the leaves. These findings revealed important differences in energy content and provitamin A value, showing that the stem is a usable part of this vegetable to be recommended in diets with low calories.

Keywords: percent composition; minerals; carotenoids; provitamin A; swiss chard.

\section{1 - INTRODUCCIÓN}

En los últimos tiempos el consumo de vegetales y hortalizas se ha difundido alentado por el incremento de dietas vegetarianas que impulsa la disminución del consumo de proteínas y grasas animales y el aumento en el aporte de fibras, minerales, vitaminas y ácidos grasos esenciales.

El contenido de nutrientes en los vegetales puede variar dentro de rangos muy amplios, dependiendo de diversos factores tales como la variedad, grado de madurez, tipo de suelo, uso de fertilizantes, intensidad y duración de la luz solar, temperatura, lluvias. En efecto, la concentración de los nutrientes depende en gran parte del contenido en minerales del suelo en que se ha cultivado o producido el alimento. Esto hace que la composición de una misma variedad cultivada en distintas regiones sea tan diferente que no permita estimar el real valor nutritivo de dichos alimentos.

Los vegetales de hojas verdes son una buena fuente de carotenoides. Estos pigmentos desempeñan un im-

1. Recebido para publicação em 09/08/2000. Aceito para publicação em 14/05/2002.

2. Instituto de Ciencias Químicas, Facultad de Agronomía y Agroindustrias, Universidad Nacional de Santiago del Estero. Av. Belgrano (s) 1912 CP: 4200. Santiago del Estero. Argentina. Fax: 0385-4222595

3. Lab. Huergo S.R.L. Perú 753. PB. CP: 1068. Capital Federal. Argentina. * A quem a correspondência deve ser enviada. portante rol en la dieta humana por su actividad provitamínica $\mathrm{A}$, sus propiedades antioxidantes y la desactivación de oxígeno singulete (el cual es mutagénico, capaz de dañar ADN, lípidos e inactivar enzimas). Las clorofilas son los pigmentos responsables del color verde de las hojas de los vegetales y de los frutos inmaduros. Son piezas claves en la fotosintesis, proceso que permite transformar la energía solar en energía química, y finalmente a partir de ella producir alimentos para todos los seres vivos y mantener el nivel de oxígeno en la atmósfera. Las plantas superiores tienen dos tipos de clorofila muy semejantes entre sí, denominadas $a$ y $b$, siendo la primera la mayoritaria y la que se degrada más fácilmente. Las clorofilas son utilizadas como aditivos alimentarios, ocasionalmente en aceites, chicles, helados y bebidas refrescantes, en sopas preparadas y en productos lácteos. También tienen la propiedad de actuar como sensibilizadores de reacciones fotoinducidas, generando oxigeno singulete.

La necesidad de la determinación de pigmentos en vegetales se debe a la importancia de los mismos por su aplicación como colorantes de alimentos así como también por los efectos benéficos para la salud que produce su consumo.

La acelga (Beta vulgaris var. cycla) es una hortaliza de hojas verdes y tallos muy engrosados, que pertenece a la familia de las Quenopodiaceas. Su cultivo en la Provincia de Santiago del Estero, Argentina, se encuentra muy di- 
fundido, especialmente la del tipo verde y blanca de penca ancha, que crece bien en toda estación y presenta alta tolerancia a la salinidad. También por su ciclo corto de crecimiento, entre 60 y 80 dias, es posible lograr varias cosechas entre abril y diciembre. A pesar de que en la provincia hay fábricas que envasan parte de la producción local, la misma sólo aprovecha la hoja de la planta sin darle uso a los tallos y su importancia económica no se encuentra registrada debido a que su consumo como alimento fresco es principalmente regional.

Los objetivos del presente trabajo fueron:

- Caracterizar en forma integral la acelga fresca tipo penca ancha producida en la región.

- Determinar el contenido de minerales, macronutrientes y pigmentos en la hoja y en el tallo de acelga para el conocer los aportes de nutrientes de este vegetal.

- Estudiar la composición de los carotenoides presentes en este vegetal para la determinación de su actividad provitamínica A.

\section{2 - MATERIALES Y MÉTODOS}

\section{1 - Muestreo y preparación de las muestras:}

Las acelgas (Beta vulgaris var. cycla) fueron recolectadas en la misma fecha (mes de agosto) en cuatro localidades de la provincia de Santiago del Estero (Argentina) formando cuatro lotes de cada localidad. Se transportaron refrigeradas después de la recolección hasta el laboratorio. Se clasificaron por tamaños, separando las que presentaban daños apreciables. Se lavaron con abundante agua bidestilada y se extendieron sobre papeles absorbentes, dejándolas secar a temperatura ambiente.

Se formaron a partir de cada lote, dos sublotes. Se separaron las hojas de los tallos, formando muestras de 100 gramos cada una. A continuación se separó el material fresco necesario para las deteminaciones que así lo requirieran (humedad, carotenoides y clorofilas). El resto se secó en estufa hasta peso constante. Una vez seco, se trituró en mortero guardando en recipientes plásticos con tapa hermética para las determinaciones posteriores.

\section{2 - Métodos Analiticos}

\section{2. 1 - Determinación de minerales}

Se determinó humedad secando las muestras en estufa a $105^{\circ} \mathrm{C}$ hasta peso constante [3]. Se mineralizó por vía seca, pesando exactamente $1,500 \mathrm{~g}$ de hoja y $2,000 \mathrm{~g}$ de tallo (1B). Se carbonizó en crisol y se llevó a mufla a $440^{\circ} \mathrm{C}$ hasta cenizas blancas. Una vez frio, se resuspendió con ácido clorhídrico concentrado (1 a 2mL) y agua desmineralizada, se calentó 10 minutos. El digerido se llevó a un volumen de $50 \mathrm{~mL}$, efectuando por duplicado las diluciones necesarias para cada mineral.

Se determinó el contenido de sodio y potasio por espectrofotometría de emisión.
Por espectrofotometría de absorción atómica (A.A.) se determinaron calcio y magnesio con llama de óxido nitroso-acetileno agregando a cada muestra 200ppm de cloruro de potasio como regulador de ionización y hierro, cinc y manganeso con llama de aire-acetileno (1B). Se utilizaron standards para A.A. Tritisol Merk.

La determinación de fósforo se realizó por el método espectrofotométrico del molibdovanadofosfato (1C) y boro por método colorimétrico del ácido carmínico [10].

Todo el material utilizado se lavó con $\mathrm{HNO}_{3}$ al 20\% enjuagándolo 3 veces con agua destilada y 3 veces con agua ultrapura. Las determinaciones se repitieron cuando los duplicados se desviaron más de un 5\% más de los valores promedios.

\section{2. 2-Determinación de constituyentes básicos}

Se realizó la determinación de fibra dietaria total por el método enzimático gravimétrico [1A], proteínas por el método de Hach, grasas totales por el método extractivo de Folch y carbohidratos totales utilizables (CHTU) por el método de la antrona de Clegg.

- Método rápido de determinación de proteínas de Hach [6]: consiste en una digestión de la muestra con ácido sulfúrico y peróxido de hidrógeno a $434^{\circ} \mathrm{C}$. Luego, se realiza una determinación colorimétrica con el reactivo de Nessler y se lee a 460nm. Se compara con una curva patrón. Luego se utiliza un factor empírico para convertir el nitrógeno en valores de proteína que es 6,25.

- Método de Folch [9A]: la fracción lipídica se extrae de la muestra por agitación vigorosa con mezcla de cloroformo/metanol a temperatura ambiente. Se agrega agua y se separa la fase orgánica. Esta se lava con solución de cloruro sódico diluida para eliminar el material proteico extraído y se deseca con sulfato sódico anhidro. El extracto clorofórmico se evapora a sequedad en un vial tarado y se pesa el residuo que contiene la grasa.

- Método de Clegg [9B]: el producto se digiere con ácido perclórico. Los almidones hidrolizados, juntamente con los azúcares solubles se determinan colorimétricamente y se expresan como glucosa.

\section{2. 3 - Determinación de carotenoides}

Se aplicó el método de RODRÍGUEZ-AMAYA et al. [12]. Los pigmentos fueron extraídos con acetona fría en una procesadora, filtrados, transferidos a éter de petróleo y concentrados en un evaporador rotatorio a $2-3 \mathrm{~mL}$. Se realizaron ensayos con saponificación en frío (hidrólisis alcalina) de los extractos etéreos para remover la clorofila, encontrándose que ésta no es necesaria y que es más conveniente no realizarla para evitar la degradación de los carotenoides durante el proceso.

Los carotenoides fueron separados sobre una columna de MgO:hyflosupercel (1:2) desarrollada con concentraciones crecientes de éter etílico o acetona en éter de petróleo. La caracterización de estos compuestos 
se basó en el orden de elución de la columna, los $R_{f}$ en cromatografia en placa delgada de sílica gel desarrolladas con dos sistemas de solventes, metanol en benceno al 3\% y benceno-acetato de etilo-metanol (75:20:5), los máximos de absorción en los espectros en dos solventes distintos y por reacciones químicas de caracterización de grupos funcionales. La cuantificación se realizó como recomienda Davies por espectrofotometría visible [5].

\section{2. 4 - Determinación de clorofilas}

Las muestras de hojas y de tallos fueron trituradas y posteriormente maceradas con el solvente de extracción, de acuerdo a lo indicado por AOAC [2]. Las clorofilas fueron extraídas con $80 \%$ de acetona en agua y filtradas. Se realizó la determinación de las concentraciones tanto de clorofilas totales como de clorofilas $a$ y $b$ en una mezcla, por espectrofotometría visible midiendo las absorbancias a 663 y $645 \mathrm{~nm}$.

Se aplicaron las ecuaciones de Arnon, usando los coeficientes de absorción específicos dados por Mackinney [7].

\section{3 - Equipamiento utilizado}

Las determinaciones por espectrofotometría de absorción atómica (A.A.) se realizaron en un espectrofotómetro de A.A. UNICAM 929. Las técnicas espectrofotométricas se llevaron a cabo en espectrofotómetros Metrolab 1600 y UNICAM UV2.

\section{4 - Justificación de la elección de los métodos}

La mineralización por vía seca presenta la ventaja frente a la digestión húmeda de que los soluciones de referencia se mantienen fácilmente en valores bajos, pudiendo tratarse grandes volúmenes de muestra cuando fuese necesario. Los experimentos de recuperación indican que, manteniendo controlada la temperatura, ninguno de los elementos medidos se pierde durante el procedimiento descripto.

Con respecto a la determinación de calcio y magnesio, dado que estos elementos pueden combinarse con otro ion presente en la muestra para formar un compuesto que se volatiliza en grado diferente que el elemento presente en los patrones (interferencia química) y que, en la llama de aire-acetileno, los alcalinotérreos son susceptibles a este tipo de interferencia, se seleccionó para su determinación la llama de óxido nitroso-acetileno. Puesto que en esta llama la ionización del calcio es importante mientras que el magnesio se ioniza en menor grado, se agregó un regulador de ionización en una concentración suficientemente alta como para suprimir la ionización del analito.

El método de Hach fue elegido para la determinación de proteínas porque la digestión se completa en 10 a 15 minutos en comparación con los métodos convencionales (Kjeldahl) que requieren de varias horas. Además no utiliza catalizadores y tanto la precisión como la exactitud, son equivalentes.
Para la determinación de grasas, aunque existen muchos métodos desarrollados para aplicaciones particulares, se escogió el método de Folch porque tiene aplicación general. La extracción con cloroformo y metanol ha demostrado poder extraer todas las principales clases de lípidos de muchos alimentos. Aunque se trata de una técnica tediosa para el trabajo rutinario tiene aplicación general.

Una de las formas de determinar el contenido de hidratos de carbono es calculando la diferencia con los otros constituyentes básicos. El método de Clegg se aplicó para poder discriminar los carbohidratos utilizables dentro de los carbohidratos totales. Por otra parte, se determinó fibra dietaria total ya que constituye un dato importante en la constitución de este tipo de alimento.

El método elegido para la determinación de boro con ácido carmínico presenta menos interferencia en comparación con el de quinalizarina (aprobado por AOAC [1], ya que las longitudes de onda de los máximos de absorción del reactivo y del complejo con el boro se encuentran más diferenciadas. Además el ácido carmínico no es sensible a pequeños cambios de temperatura a diferencia de la quinalizarina.

En cuanto al análisis de los carotenoides para evaluar su valor como provitamina A, se seleccionó el método de Rodriguez-Amaya por considerarlo más adecuado que los recomendados por la AOAC [4] y por COST 91 (European Cooperation in Scientific and Technological Research) [11]. Estos últimos son cuantitativos pero no discriminan entre los distintos pigmentos aún cuando estos presentan diferencias en sus actividades provitamínicas. Además, se considera relevante la diferenciación de carotenoides, ya que algunos, si bien no son provitamínicos, también presentan efectos benéficos para la salud por su carácter antioxidante [8].

\section{3 - RESULTADOS Y DISCUSIÓN}

\section{1 - Constituyentes básicos}

Los resultados obtenidos en las determinaciones realizadas se muestran en la Tabla 1.

TABLA 1. Composición Centesimal de Acelga ${ }^{a}$

\begin{tabular}{lcc}
\hline Determinación & Hoja (\%) & Tallo (\%) \\
\hline Proteínas & $2,9 \pm 0,3$ & $1,2 \pm 0,1$ \\
Grasas & $0,17 \pm 0,04$ & $0,09 \pm 0,01$ \\
Cenizas & $2,0 \pm 0,2$ & $1,3 \pm 0,1$ \\
Carbohidratos utilizables & $0,4 \pm 0,1$ & $0,56 \pm 0,09$ \\
Fibra Dietaria total & $3,9 \pm 0,2$ & $2,9 \pm 0,2$ \\
Humedad & $90,6 \pm 0,7$ & $94 \pm 1$ \\
\hline
\end{tabular}

a Valores promedio y desviaciones standard.

Se puede observar que la hoja contiene mayor cantidad de proteinas, grasas, cenizas y fibra dietaria total que el tallo mientras que éste contiene mayor cantidad de 
hidratos de carbono utilizables y humedad que la hoja. Estas proporciones varían considerablemente si se refiere a peso seco de la muestra, debido al gran porcentaje de humedad presente en los tallos. En estos, el porcentaje de extracto seco es $6,02 \mathrm{~g}$, por lo tanto, la cantidad de fibra en base seca es $48,00 \%$ mientras que en la hoja cuyo extracto seco es $9,41 \%$, la fibra dietaria es $41,12 \%$. A partir de estos resultados se puede establecer que la fibra es realmente el principal constituyente de este vegetal.

De acuerdo a los valores obtenidos de los constituyentes se realizó el cálculo de Contenido Energético obteniéndose los siguientes resultados: Hoja: $14,91 \mathrm{Kcal} / \mathrm{g}$ y Tallo: $8,07 \mathrm{Kcal} / \mathrm{g}$.

\section{2- Minerales}

Los resultados obtenidos en la determinación de los minerales se muestran en la Tabla 2. Del análisis comparativo del contenido de minerales en tallo y hoja se desprende que existen cantidades similares de potasio. Los contenidos de fósforo, calcio, sodio, magnesio y boro son aproximadamente el doble en la hoja con respecto al tallo, mientras que para hierro, manganeso y zinc, es de cuatro a cinco veces superior.

TABLA 2. Contenido de Minerales en Hojas y Tallos de Acelga ${ }^{a}$

\begin{tabular}{ccc}
\hline $\begin{array}{c}\text { Minerales } \\
(\mathrm{mg} / 100 \mathrm{~g})\end{array}$ & Hoja & Tallo \\
\hline $\mathrm{Ca}$ & $101 \pm 8$ & $53 \pm 5$ \\
$\mathrm{Mg}$ & $52 \pm 3$ & $30 \pm 2$ \\
$\mathrm{Na}$ & $235 \pm 14$ & $150 \pm 8$ \\
$\mathrm{~K}$ & $493 \pm 19$ & $470 \pm 30$ \\
$\mathrm{Mn}$ & $3.3 \pm 0.3$ & $0.63 \pm 0.06$ \\
$\mathrm{Zn}$ & $0.39 \pm 0.03$ & $0.10 \pm 0.05$ \\
$\mathrm{Fe}$ & $1.8 \pm 0.3$ & $0.34 \pm 0.06$ \\
$\mathrm{P}$ & $41 \pm 5$ & $23 \pm 4$ \\
$\mathrm{~B}^{\mathrm{b}}$ & $40 \pm 4$ & $16 \pm 3$ \\
\hline
\end{tabular}

${ }^{a}$ Valores promedio y desviaciones standard. ${ }^{b}$ Expresado en $\mu \mathrm{g} / 100 \mathrm{~g}$.

\section{3 - Carotenoides}

\section{3. 1 - Aislación, identificación y cuantificación}

En las hojas de acelga fueron aislados cuatro carotenoides por cromatografia en columna abierta de MgO-Hyflosupercel (1:2): $\beta$-caroteno, $\alpha$-caroteno, luteína y violaxantina. La primera fracción obtenida de la columna fue eluída con $2-6 \%$ de éter etílico en éter de petróleo. El comportamiento cromatográfico y el espectro UV-Visible en éter de petróleo son típicos de $\alpha$-caroteno según se observa en la Tabla 3. La ausencia de sustituyentes fue demostrada por TLC sobre placas de sílica gel desarrolladas con éter de petróleo $\left(R_{F}=0,33\right)$ y $3 \%$ de metanol en benceno $\left(R_{F}=0,94\right)$, mientras que la ausencia de grupos epóxidos se determinó por la falta de cambio de color de la mancha por la exposición a los vapores de $\mathrm{HCl}$.

La segunda fracción eluída de la columna con 6-10\% de éter etílico en éter de petróleo presenta los máximos de absorción característicos de $\beta$-caroteno. $E 1 R_{\mathrm{F}}$ sobre placa de sílicagel desarrollada con éter de petróleo fue 0,32 y en $3 \%$ de metanol en benceno el $R_{F}$ fue de 0,94 lo que confirma la presencia de un compuesto sin sustituyentes hidroxílicos. La ausencia de grupos epóxidos fue demostrada como en la primera fracción y es consistente con la identificación realizada.

TABLA 3. Máximos de Absorción en los espectros UV-visible de los carotenoides extraídos de acelga.

\begin{tabular}{ccr}
\hline Fracción & Carotenoides & \multicolumn{1}{c}{$\lambda_{\max } \mathrm{UV}-\mathrm{Vis}$} \\
\hline 1 & $\alpha$-carotene & $419-442-471^{\mathrm{a}}$ \\
2 & all-trans $-\beta$-carotene & $447-474^{\mathrm{a}}$ \\
$3-1$ & luteína & $421-444-472^{\mathrm{b}}$ \\
$3-2$ & violaxantina & $419-440-470^{\mathrm{b}}$ \\
\hline
\end{tabular}

${ }^{\mathrm{a}}$ Determinados en éter de petroleo. ${ }^{\mathrm{b}}$ Determinados en etanol.

La tercera fracción eluída con $20 \%$ de acetona en éter de petróleo, fue recromatografiada en columna de alúmina neutra, obteniéndose dos fracciones. La primera fracción (3-1) mostró valores de $R_{F}$ de 0,20 en placas de sílicagel desarrolladas con $3 \%$ de metanol en benceno y $R_{\mathrm{F}}$ de 0,63 usando como solvente de elución una mezcla de 75:20:5 benceno-acetato de etilo-metanol. No se observó cambio de color de la mancha por la exposición a los vapores de $\mathrm{HCl}$. Los máximos de absorción son característicos de un cromóforo similar al de $\alpha$-caroteno y no presentaron corrimientos por la adición de $\mathrm{HCl}$. La presencia de grupos hidroxilos (primarios o secundarios) y en posición alílica fue determinada por la respuesta positiva a la acetilación y a la metilación, respectivamente. Esta fracción fue identificada como luteína ( $\beta$, $\varepsilon$-caroteno-3,3-diol) por las evidencias antes mencionadas.

La otra fracción (3-2) con 15\% de acetona en éter de petróleo, dio un valor de $R_{F}$ de 0,11 sobre placas de sílicagel desarrolladas con $3 \%$ de metanol en benceno y cuando se eluyó con (75:20:5) de benceno-acetato de etilometanol el valor de $R_{\mathrm{F}}$ fue de 0,42 , tornándose la mancha de color azul por la exposición a los vapores de $\mathrm{HCl}$. La presencia de grupos hidróxilos (primarios $\mathrm{o}$ secundarios) fue determinada por la respuesta positiva a la acetilación. La existencia de dos grupos epóxidos se determinó por el corrimiento hipsocrómico de $40 \mathrm{~nm}$ de los máximos de absorción en el espectro en etanol con la adición de $\mathrm{HCl}$ al $20 \%$. Esta fracción fue identificada como

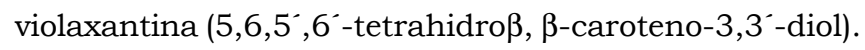

Los resultados obtenidos en la cuantificación de los carotenoides se informan en la Tabla 4.

De acuerdo lo informado en la literatura, los tejidos verdes de plantas superiores contienen como carotenoides principales, $\beta$-caroteno, luteína, violaxantina y neoxantina mientras que a-caroteno puede aparecer en pequeñas cantidades [13]. Los pigmentos encontrados en hojas de acelga coinciden con los esperados sobre la base de los 
antecedentes para vegetales de hojas verdes [14], aunque no se aisló neoxantina.

TABLA 4. Contenido de Carotenoides de Acelga

\begin{tabular}{lccccc}
\hline $\begin{array}{l}\text { Concentración } \\
(\mu \mathbf{g} / \mathbf{g})^{\mathbf{b}}\end{array}$ & $\alpha$-caroteno & $\beta$-caroteno & luteína & violaxantina & Total \\
\hline Hojas & $0,35 \pm 0,04$ & $27 \pm 1$ & $27 \pm 1$ & $9,2 \pm 0,9$ & $64 \pm 2$ \\
Tallos & - & - & - & - & $0,14 \pm 0,09^{\mathbf{a}}$ \\
\hline
\end{tabular}

apuesto que la concentración de carotenoides observada en los tallos es muy baja, a diferencia de lo realizado en las hojas, no se realizó la determinación individual. b Valores medios y desviaciones standard para 3 muestras analizadas.

\section{3. 2 - Valores de vitamina A}

La cuantificación del valor de vitamina A se realizó sobre la base de la actividad provitaminica individual para cada carotenoide. El valor de vitamina A de las hojas estudiadas es de 9,12 Eq. totales de vitamina A/g y 454 Eq retinol/100g. El valor vitamínico A de los tallos es prácticamente despreciable. En lo referido a la ingesta diaria recomendada, para cubrir esta necesidad, considerándose un valor promedio de $800 \mathrm{~g}$ Vit.A/día, se deberían consumir $176 \mathrm{~g}$ de hojas de acelga (fresca) por día.

\section{4 - Clorofilas}

Las clorofilas fueron identificadas por sus espectros típicos de absorción con una banda entre 650 a $700 \mathrm{~nm}$ y otra a 430nm caracteristica de clorofila a y a 450 y $465 \mathrm{~nm}$ correspondiente a clorofila $b$.

La cuantificación se realizó basándose en las ecuaciones de Arnon [7]. Los resultados obtenidos son informados en la Tabla 5.

TABLA 5. Contenido de Clorofilas

\begin{tabular}{cccc}
\hline $\begin{array}{c}\text { Concentración } \\
\text { a } \\
(\mathbf{m g} / \mathbf{g})\end{array}$ & Clorofila a & Clorofila b & Total \\
\hline Hojas & $0,66 \pm 0,02$ & $0,31 \pm 0,01$ & $0,96 \pm 0,02$ \\
Tallos & $0,088 \pm 0,003$ & $0,084 \pm 0,003$ & $0,172 \pm 0,004$ \\
\hline
\end{tabular}

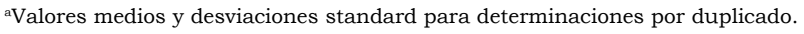

\section{4 - CONCLUSIONES}

Se comprobaron diferencias en el aporte energético, minerales, proteínas, grasa y fibras entre hoja y tallo, siendo preponderante la concentración de los mismos en la hoja del vegetal mientras que para hidratos de carbono y potasio se observaron valores similares. Esta diferencia se ve resaltada por el elevado contenido de agua de los tallos ya que si expresáramos su composición en peso seco de muestra, esta brecha sería menor y hasta en algunos casos se revertiría el predominio.

Estas características hacen de la acelga un alimento de bajo contenido energético, apto en su integridad para el consumo humano y excelente alternativa para dietas vegetarianas y para aquellas que buscan incrementar la ingesta de alimentos de bajas calorías, con un importante aporte de carotenoides (provitaminas A y antioxidantes), fibra y minerales, especialmente en potasio y boro donde cubre una buena parte de la ingesta diaria recomendada.

\section{5 - REFERENCIAS}

[1] ASSOCIATION OF OFFICIAL ANALYTICAL CHEMISTS. Official Methods of Analysis of the A.O.A.C. $15^{\text {th }}$ Ed. Washington, D.C. US Government Printing Office, 1990. A) Total dietary fiber in foods. Enzymatic Gravimetic Method. (985.29). B) Metals in plants: Atomic Absorption Spectrophotometric method. (975.03). C) Phosphorous (total) in fertilizers. Spectrophotometric molybdovanadophosphate method. (958.01).

[2] ASSOCIATION OF OFFICIAL ANALYTICAL CHEMISTS. Official Methods of Analysis of the A.O.A.C. $11^{\text {th }} \mathrm{Ed}$. Washington, D.C. US Government Printing Office, 1970.

[3] ASSOCIATION OF OFFICIAL ANALYTICAL CHEMISTS. Official Methods of Analysis of the A.O.A.C. $12^{\text {th }} \mathrm{Ed}$. Washington, D.C. US Government Printing Office, 1985.

[4] ASSOCIATION OF OFFICIAL ANALYTICAL CHEMISTS. Official Methods of Analysis of the A.O.A.C. $14^{\text {th }} \mathrm{Ed}$. Arlington, VA, 834, 1984.

[5] DAVIES, B. H. Carotenoids in Chemistry and Biochemisty of Plant Pigments. T. W. GOODWIN, $2^{\text {nd }}$ Ed., Academic Press, London, v.2, p.38-165, 1976.

[6] HACH , C. C.; BRAYTON, S. V.; KOPELOVE, A.B. A powerfull Kjeldahl nitrogen method using peroxy monosulfuric acid. J. Agric. Food Chem. v.33, p.1117-1123, 1985.

[7] HOLDEN, M. Chlorophylls in Chemistry and Biochemisty of Plant Pigments. T. W. GOODWIN, $2^{\text {nd }}$ Ed., Academic Press, London, v.2, p. 2-37, 1976.

[8] KRINSKY, N. I. Antioxidant Functions of Carotenoids in Free Radical Biology \& Medicine. v.7, p. 617-637, 1989.

[9] OSBORNE, D.R., VOOGT, P. Análisis de los nutrientes de los alimentos. Ed. Acribia. Zaragoza, España, 1978. A) Determinación de grasa total. Método de extracción con cloroformo-metanol (Folch), p.168-170. B) Carbohidrato utilizable total (método manual de la antrona de Clegg). p.136-137.

[10] PORTER, G. AND SHUBERT, R. in Chemical Analysis. Colorimetric determination of nonmetals. BLACK, S. AND BOLTZ, D. Editor: David Boltz, London, p. 339353, 1958.

[11] RAMOS, D. M.; RODRIGUEZ- AMAYA, D. B. Determination of the vitamin A value of common Brazilian leafy vegetables. J. Micronutrient Anal. v. 3, p.147-155, 1987.

[12] RODRIGUEZ-AMAYA, D.B. et al. Carotenoid pigment changes in ripening Momordica charantia fruits. Ann. Bot., v. 40, p.615-624, 1976.

[13] GOODWIN, T. W. Distribution of Carotenoids in Chemistry and Biochemisty of Plant Pigments. T. W. GOODWIN, $2^{\text {nd }}$ Ed., Academic Press, London, v.2, p.225-257, 1976.

[14] MERCADANTE, A., RODRIGUEZ-AMAYA, D. Carotenoid composition and vitamin A value of some native Brazilian green leafy vegetables. Intern. J. of Science and Tech., 1989.

\section{6 - AGRADECIMIENTOS}

Se agradece al Ing. Domingo Rosas por su colaboración en la determinación de proteínas.

Este trabajo de investigación fue posible por el apoyo financiero otorgado por el CICyT-UNSE, ANPCyT y Fundación Antorchas. 